\title{
Wigner Distributions and Phase Space in Optics
}

\section{INTRODUCTION}

Our call for papers on Wigner distributions and phase space in optics received a hearty response. In this feature issue we have gathered papers that show, from quite different perspectives, the relevance and promise of phase space for optics. It was satisfying to receive such a range of viewpoints on the principal object of attention: quasiprobability distributions on phase space, i.e., Wigner functions. The phase-space function defined by Eugene Wigner on statistical grounds for quantum mechanics has proved to be a remarkably pliable tool in optics. In quantum optics, in fact, phase space is the canvas depicting the very nature of light. However, it is also clear that other, closely related, ideas play key roles in a variety of phase-space methods for optics.

Given the interdisciplinary character of this research, we somewhat arbitrarily have grouped papers from the abstract to the concrete, and we have further subdivided the latter. Within each group papers appear in alphabetical order. The reader will appreciate from the references of these papers how lines of disparate research have converged. In contrast to the melding of space and time in relativity theory, the fusing of coordinate and frequency domains to form optical phase space has been a slower, more incremental process. As becomes clear from this collection, this process has been spurred in part by studies of short pulses, of image and information processing, and of semiclassical issues related to the raywave connection in various domains of wave physics.

A number of fundamental issues arise in several of the papers. Localization, for example, is a central notion in phase space, together with the well-known uncertainty relation, which sets a lower bound on the products of the moments of the Wigner distribution. Generalized uncertainty relations are also discussed here, as is their connection to various types of nonclassical states of light (such as squeezed and correlated states) and to beam purity measures. Similarly, the fractional Fourier transfor- mation has received considerable interest since the early nineties, especially in the signal processing and optics communities, and it arises repeatedly in these papers. The Wigner distribution of the fractional Fourier transform is a rotated version of the Wigner distribution of the original function. Furthermore, the integral projection of the Wigner distribution onto any oblique axis in phase space yields the squared magnitude of the associated fractional Fourier transform of the function. It is not surprising, therefore, that the fractional Fourier transform is so widely used here. For the analysis of discrete signals, several of the papers propose discrete analogs of this and other transforms that act on models of discrete phase spaces. Another unifying theme relates to the Gaussian profiles that couple coherent-state representations, Gabor transforms, and windowed Fourier transforms, among other spectrograms.

We hope that, given the breadth of this research, most of the readers of the Journal of the Optical Society of America $A$ will find in these papers insights and gems that spark their own ideas and interests. The topic featured in this special issue is so evidently a rich field, and it continues to be enriched by the efforts of many researchers, including the authors of these papers. We are grateful for their contributions to this collection and to the staff at the OSA manuscript office, who were also essential to its creation.

\author{
Gregory William Forbes \\ V. I. Man'ko \\ Haldun M. Ozaktas \\ R. Simon \\ Kurt Bernardo Wolf \\ Feature Editors \\ Wigner Distributions and \\ Phase Space in Optics
}

